\title{
Commentary on "Clinical Characteristics and Adequate Treatment of Familial Adenomatous Polyposis Combined with Desmoid Tumors"
}

\author{
Edoardo Virgilio, MD', Francesca Di Gregorio, MD², Genoveffa Balducci, MD' \\ ${ }^{1}$ Medical and Surgical Sciences and Translational Medicine, \\ ${ }^{2}$ Department of Radiology, Faculty of Medicine and Psychology "Sapienza", St. Andrea Hospital, Rome, Italy
}

We commend Jung et al. [1] on their noteworthy effort to describe the clinicopathological features, risk factors, and outcomes of familial adenomatous polyposis (FAP) patients with desmoid tumors (DTs). In this regard, we would like to stress a particular and disturbing aspect of this disease, which is the potentially ambiguous role of surgery towards young FAP patients with colorectal cancer (CRC). In fact, in these patients, the post-surgical development of massive intra-abdominal DTs represents a fatal event in the vast majority of cases, as discussed in more detail hereafter.

Recently, we performed total proctocolectomy in a 28-year-old nulliparous FAP-girl (APC mutation 3' to codon 3238) who developed a cancerous polyp of the rectum. The patient did well after the intervention until, after 6 months, she developed a pregnant looking abdomen, even though she was not pregnant. A computed tomography scan showed a solid mass of the mesentery measuring $28 \mathrm{~cm} \times 21 \mathrm{~cm}$, which occupied the entire abdominal cavity encasing the superior mesenteric vessels and both common iliac arteries: a radiological diagnosis of giant mesenteric DT was notched (Fig. 1). Due to the irresectable nature of the lesion, the patient was referred to a specialized center for intestinal and multivisceral transplantation, but, during evaluation she became rapidly aggravated until death.

Despite being extremely rare in the general population, intra-abdominal DTs (also known as mesenteric fibromatosis) represent the most frequent cause of death in patients affected with FAP having undergone total proctocolectomy [2]. Although histologically benign and clinically non-metastasizing, these monoclonal myofibroblastic proliferations can show a highly aggressive local behavior, achieving massive size, infiltrating major vessels and causing severe complications, such as bowel ischemia and obstruction, fistulae with peritonitis, bleeding and venous thromboembolism [2]. Young nulliparous women using contraceptives with history of colectomy are at higher risk of development [2]. Pathobiology is poorly understood: consequently, their behavior is unpredictable and there is no standardized medical or surgical treatment [1]. A valuable measure in management of this kind of lesion was provided in 2005 by Church et al. [3] who proposed a clinical staging system
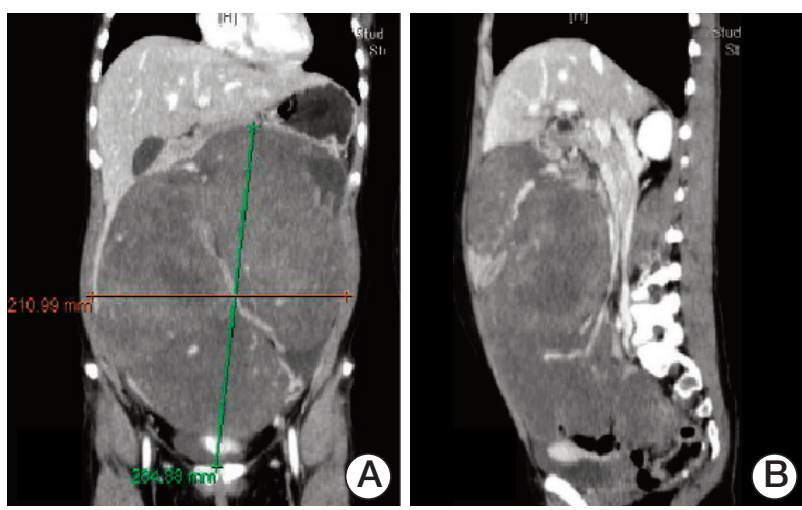

Fig. 1. Coronal (A) and sagittal (B) view of the stage IV intra-abdominal desmoid tumor occupying the entire abdominal cavity and encasing vital vascular structures. based on tumor size, growth and the presence of symptoms and complications. While stage I, II, and III are amenable to several modalities of treatment (antiestrogens, nonsteroidal anti-inflammatory drugs, selective tyrosine kinase inhibitor, chemotherapy, and surgical resection), stage IV tumors (larger than $20 \mathrm{~cm}$, rapidly growing and severely symptomatic such as our case) are characterized by higher morbidity and mortality rates: indeed, any medical treatment seems to be in vain as too much time is required for any type of benefit and surgical resection is often impossible for the encasement of vital vessels [1]. Currently, advanced unresectable mesenteric DTs are the fourth most common indication for intestinal transplant intervention worldwide [4]. However, not all of these patients arrive at transplantation (as in our case) and the 5-year survival rate does not exceed 70\% [2]. Greater knowledge of giant inoperable mesenteric DTs is needed, and, in particular, young FAP patients should be thoroughly informed on the risk of this ominous event when undergoing colectomy for CRC and carefully followed-up.

Correspondence: Edoardo Virgilio, MD

Medical and Surgical Sciences and Translational Medicine, Faculty of Medicine and Psychology "Sapienza", St. Andrea Hospital, via di Grottarossa 1035-39, Rome 00189, Italy Tel: 39-633775989, Fax: 39-633775322, E-mail: aresedo1992@yahoo.it

Received February 2, 2015 Accepted February 12, 2015 Published online February 26, 2015 


\section{Conflicts of Interest}

Conflict of interest relevant to this article was not reported.

\section{References}

1. Jung WB, Kim CW, Kim JC. Clinical characteristics and adequate treatment of familial adenomatous polyposis combined with desmoid tumors. Cancer Res Treat. 2014;46:366-73.

2. Chaudhary P. Mesenteric fibromatosis. Int J Colorectal Dis. 2014;29:1445-51.

3. Church J, Berk T, Boman BM, Guillem J, Lynch C, Lynch P, et al. Staging intra-abdominal desmoid tumors in familial adenomatous polyposis: a search for a uniform approach to a troubling disease. Dis Colon Rectum. 2005;48:1528-34.

4. Quintini C, Ward G, Shatnawei A, Xhaja X, Hashimoto K, Steiger E, et al. Mortality of intra-abdominal desmoid tumors in patients with familial adenomatous polyposis: a single center review of 154 patients. Ann Surg. 2012;255:511-6. 